\title{
EFFECTS OF EMPIRICAL DISSIPATION TERMS IN THE SOLUTION OF THE UNDULAR BORE*
}

\author{
BY \\ JOHN G. B. BYATT-SMITH \\ University of California at Berkeley
}

\begin{abstract}
In this paper we consider the problem of a steady bore running downhill. The effects of dissipation are included empirically, using the Chézy law. The method of solution is based on an averaging technique which assumes that the uniform solution is slowly varying.

1. Introduction. The study of the finite amplitude effects upon long waves in channels was started as long ago as 1845 by Airy [1]. From the first investigations emerged the fact that long waves must change their form as they advance, becoming steeper ahead of their peaks and less steep behind. Such steeping would, in the absence of any other tendency, lead to the appearance of bores of hydraulic jumps. However, the experimental discovery of the solitary wave (Russell, 1837, 1844 [12]) and later the theoretical investigations of de Boussinesq and Lord Rayleigh, 1876 [11], threw doubts on the truth of the original investigation. This led to the final elucidation of long waves by Korteweg and De Vries in 1895 [8]. They showed that permanent, finite amplitude waves were possible and termed them cnoidal waves.

The classical theory of the bore (Rayleigh, 1914 [11]) is based on a transition between two uniform flows through which mass and momentum flux is conserved. Although no loss of momentum due to frictional forces at the bottom is considered, the solutions show that energy must be lost at the bore, and it was suggested that this was due to frictional dissipation or turbulence.

For a strong bore it is generally accepted that this energy loss occurs by breaking and turbulence just downstream of the bore. But it is found experimentally that weak bores have a stationary train of waves behind them and exhibit no tendency to break (Favre, 1935 [6]). Lemoine [9] quoting these results suggests that under these circumstances the required energy loss may occur by radiation through the wave train. Lemoine assumed the waves were sinusoidal and calculated their amplitude and the resulting rate of radiation of energy through them.

His results were, however, only in moderate agreement with experiments. This led Benjamin and Lighthill to doubt the fact that the wave train was sinusoidal. They decided to follow the suggestion of Keulegan and Patterson [7] that the wave train was cnoidal. In their work they defined three quantities, $Q$ the volume flow rate, $R$ the energy, and $S$ the momentum flow rate. In the absence of friction these quantities remained constant for each cross-section of the flow. They also showed that it was possible to match a steady train of cnoidal waves downstream to a uniform upstream
\end{abstract}

${ }^{*}$ Received October 8, 1969. 
flow only if there was a change in either $Q, R$, or $S$. For cases where $Q$ and $S$ remained constant they related the resulting wave train behind the bore to the amount by which $R$ was reduced, varying from waves of large wavelength for very little loss of energy to waves of very small wavelength when the loss of energy is that given by the classical theory.

Since the publication of the work by Benjamin and Lighthill [2], it was clear that further research into the problem would have to include the dissipation effects of, for example, friction or turbulence. Although very little has been done to include the effects of a boundary layer at the bottom the effects of empirical dissipation terms have been studied by various authors for almost a century. This early treatment, however, takes only the Airy approximations for long waves and an empirical friction law, such as the Chézy Law. The only steady profiles that were produced were monoclinical flood waves. Such calculations of the slope of the profile have been given by Thomas [15] and Dressler [5]. Dressler also looked for a steady periodic solution but found he had to introduce discontinuities or bores. This steady solution occurs when the basic flow is unstable and breaks down into a series of roll waves. The theory was revised by Lighthill and Whitham in 1955 [10]. They derived a general theory of kinematic waves and took as one example of their theory the flood movement of rivers.

The first quantitative discussions of the effects of friction on cnoidal waves appears to have been done by Sandover and Zienkiewicz in 1957 [14], when they studied an undular surge wave entering still water in a rectangular channel. They assumed that after a sufficient time the profile was steady and that the changes in energy and momentum were given by the Chézy Law. This was repeated and extended to channeis of trapezoidal cross-sections by Sandover and Taylor in 1962 [13]. The resulting ordinary differential equation was integrated numerically. Their results were in very good agreement with experiment for the leading waves, but the amplitude of the oscillations they obtained behind the bore was not damped out and did, in fact, grow as they integrated downstream. This they suggested was due to the fact that no allowance was made for the effects of breaking. Of course, if the bore was very strong and breaking did occur then these effects would not be accounted for by the empirical terms of Sandover and Taylor, and Sandover and Zienkiewicz. However, it is difficult to see why their solutions should not give a good result when the bore is very weak so that no breaking would be expected.

In this paper we will investigate the effect of introducing the empirical Chézy Law frictional terms to see if a steady solution is possible.

\section{Derivation of equations.}

2.1 The basic equations. The basic equations for the mean velocity $\bar{u}$ and the height $h$ are obtained by integrating the Navier-Stokes equations across the crosssection of the channel. To obtain a solution it is necessary to include a body force in the direction of the flow to counteract the effects of the dissipation forces. The flow is therefore assumed to take place down a slightly inclined bed, as indicated by Fig. 1. The coordinate axes are then taken along and perpendicular to the channel bed.

The equations of motion are then

$$
\frac{\partial u}{\partial x}+\frac{\partial v}{\partial y}=0,
$$




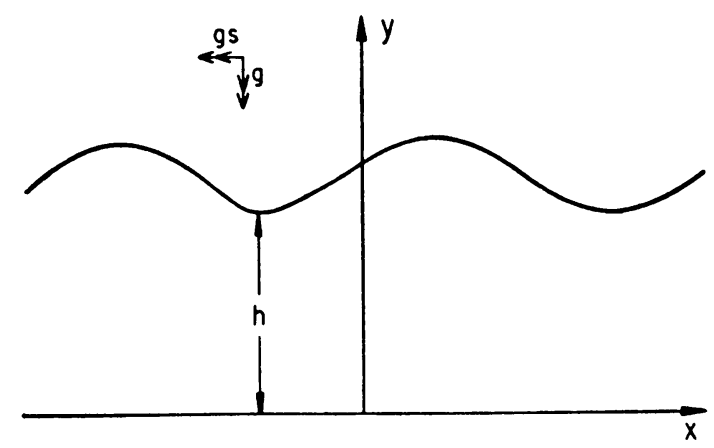

FIg. 1. The coordinate axes

$$
\begin{gathered}
\frac{\partial u}{\partial t}+u \frac{\partial u}{\partial x}+v \frac{\partial u}{\partial y}=-\frac{1}{\rho} \frac{\partial p}{\partial x}-g s+f, \\
\frac{\partial v}{\partial t}+u \frac{\partial v}{\partial x}+v \frac{\partial v}{\partial y}=-\frac{1}{\rho} \frac{\partial p}{\partial y}-g .
\end{gathered}
$$

The first equation is the equation of continuity; the second is the usual $x$-momentum equation with the added body force $f$, and the last is the usual $y$-momentum equation.

We also have the boundary condition at the free surface $y=h$

$$
\frac{\partial h}{\partial t}+u \frac{\partial h}{\partial x}-v=0 \text {. }
$$

Using the continuity equation this gives

$$
\frac{\partial h}{\partial t}+\frac{\partial}{\partial x}\left(\int_{0}^{h} u d y\right)=0
$$

If we define the mean velocity $\bar{u}$ by

$$
\bar{u}=\frac{1}{h} \int_{0}^{h} u d y,
$$

then Eq. (2.1.4) can be written as

$$
\frac{\partial h}{\partial t}+\frac{\partial}{\partial x}(\bar{u} h)=0 .
$$

With the aid of Eq. (2.1.4) integration of Eq. (2.1.2) across the cross-section gives

$$
\frac{\partial \bar{u}}{\partial t}+\bar{u} \frac{\partial \bar{u}}{\partial x}+\frac{1}{h} \frac{\partial}{\partial x} \int_{0}^{h}(\bar{u}-u)^{2} d y=\frac{1}{h} \int_{0}^{h}\left(-\frac{1}{\rho} \frac{\partial p}{\partial x}+f\right) d y-g s .
$$

We now appeal to a long wave theory and an order of magnitude agreement to neglect the integral appearing on the left-hand side of Eq. (2.1.8). On a long wave theory if $U_{0}$ (a constant) is a typical velocity scale and $a$ is a typical amplitude scale, then $(\bar{u}-u)$ is of order $U_{0}^{2} a^{2}$ so that the integrant is of order $U_{0}^{4} a^{4}$ and can be neglected.

Thus Eq. (2.1.8) becomes

$$
\frac{\partial \bar{u}}{\partial t}+\bar{u} \frac{\partial \bar{u}}{\partial x}=\frac{1}{h} \int_{0}^{h}-\frac{1}{\rho} \frac{\partial p}{\partial x} d y+\bar{f}-g s,
$$

where $\bar{f}=(1 / h) \int_{0}^{h} f d y$ is the averaged body force due to dissipation. 
2.2 The evaluation of the pressure. To evaluate the pressure we proceed on the basis that the dispersion effect is small and can be accounted for on a two-dimensional linear theory with dissipation neglected. The analysis has been given by Chester [4], and need not be repeated here. His result is

$$
\frac{1}{h} \int_{0}^{h} \frac{1}{\rho} \frac{\partial p}{\partial x} d y=g \frac{\partial h}{\partial x}+\frac{g h_{0}^{2}}{3} \frac{\partial^{3} h}{\partial x^{3}}+\cdots,
$$

where $h_{0}$ is the undisturbed height.

We approximate by keeping only the first two terms.

When this is substituted into Eq. (2.1.9) we obtain as our basic equations

$$
\begin{gathered}
\frac{\partial \bar{u}}{\partial t}+\bar{u} \frac{\partial \bar{u}}{\partial x}+g \frac{\partial h}{\partial x}+\frac{g h_{0}^{2}}{3} \frac{\partial^{3} h}{\partial x^{3}}=\bar{f}-g s, \\
\frac{\partial h}{\partial t}+\frac{\partial}{\partial x}(\bar{u} h)=0 .
\end{gathered}
$$

A steady solution is found by looking for a solution where $u$ and $h$ are functions of the single variable

$$
\xi=x+U t
$$

where $U$ is the velocity of the profile relative to the fixed axes.

Eq. (2.2.3) can then be integrated to give

$$
(\bar{u}+U) h=Q .
$$

Then elimination of $\bar{u}$ gives to our order of approximations

$$
\frac{g h_{0}^{2}}{3} \frac{\partial^{3} h}{\partial \xi^{3}}+\frac{\partial h}{\partial \xi}\left(g-\frac{Q^{2}}{h^{3}}\right)=\bar{f}-g s .
$$

This can be nondimensionalized by setting

$$
h=h_{0}(1+\eta), \quad \xi=h_{0} X .
$$

The equation for $\eta$ is then

$$
\frac{1}{3} \eta^{\prime \prime \prime}+3 \eta \eta^{\prime}-\left(F^{2}-1\right) \eta^{\prime}=\frac{\bar{f}}{g}-S,
$$

In Eq. (2.2.6) $F=Q /\left(g h_{0}^{3}\right)^{1 / 2}$ and terms of order $\eta^{3}$ are neglected.

2.3 The dissipational bocly force $\bar{f}$. If the dissipation is given by the Chézy Law, then, this assumes that the total force over a cross-section due to dissipation is proportional to the square of the mean velocity. Thus

$$
\int_{0}^{h} f d y=C^{2} \bar{u}^{2}
$$

where $C$ is some constant.

Using the value for $\bar{u}$ from Eq. (2.2.4), we obtain

$$
\bar{f}=\frac{g C^{2}\left[F_{1}(1+\eta)-F\right]^{2}}{(1+\eta)^{3}}
$$

where $F_{1}=u\left(g h_{0}\right)^{-1 / 2}$. Thus 


$$
\frac{\bar{f}}{g}-S=-S+\frac{C^{2}\left(F_{1}(1+\eta)-F\right)^{2}}{(1+\eta)^{3}}
$$

since $h_{0}$ was the undisturbed height $\eta \rightarrow 0$ as $x \rightarrow-\infty$. Therefore, $F_{1}=F+\sqrt{ } S / C$,

$$
\frac{\bar{f}}{g}-S=\frac{-S\left[\eta^{3}+\left(2-2 F_{2}+F_{2}^{2}\right) \eta^{2}-\left(2 F_{2}-1\right) \eta\right]}{\left(1+\eta^{3}\right)}
$$

where $F_{2}=C F_{1} / \sqrt{ } S$. For flows between subcritical and supercritical states we must have $F>1$ and $F_{2}>\frac{1}{2}$. In this case the right-hand side of Eq. (2.3.4) can be approximated to $S\left(\eta_{2}-\eta\right) \eta$.

Therefore Eq. (2.2.6) becomes

$$
\frac{1}{3} \eta^{\prime \prime}+3 \eta \eta^{\prime}-\left(F^{2}-1\right) \eta^{\prime}=S\left(\eta_{2}-\eta\right) \eta .
$$

This equation can be integrated and, as Sandover and Taylor and Sandover and Zienkiewicz found, the leading waves were of the general form expected from experimental observations, but the solution did not settle down to $\eta_{2}$ as $x \rightarrow \infty$. In fact, if we linearize about $\eta_{2}$, then the solution is a sine wave with an exponentially growing amplitude as $x \rightarrow \infty$. Thus when we introduce the Chézy term, we cannot hope to obtain a solution that tends to the uniform flow solution $\eta=\eta_{2}$. Hence we are led to the question-does a solution of Eq. (2.3.5) exist which is bounded for all $x$ ? The answer to this quation is provided by the method of averaging.

\section{Method of averaging.}

3.1 Introduction to the method of averaging. In this section we take up the problem of finding approximate analytic solutions to the periodic solutions of Eq. (2.3.5) in the case when $S$ is small. From the physical volume of the problem described by Eq. (2.3.5) no dissipation corresponds to zero $S$ and in this case the solution will be cnoidal waves of constant amplitude. If $S$ is small the length scale $\left(0 S^{-1}\right)$ which characterizes the dissipative processes is very much larger than the length scale or wavelength of the cnoidal solutions. Care must be taken here when assuming that the wavelength of the cnoidal solution is of the same order of magnitude as that of a typical length scale of the cnoidal solutions, because for the solitary wave the wavelength becomes infinite. However, this is misleading, since an effective length scale for the solitary wave can easily be defined. 'This point is made and discussed by Benjamin and Lighthill ([2], 1954). Thus during one oscillation the dissipation can produce only a small effect and we can anticipate that the amplitude and phase of the oscillations will change only very gradually. Consequently, we can allow for the effect of the dissipation terms by calculating their average over a wavelength.

Eq. (2.3.5) is

$$
\frac{1}{3} \frac{d^{3} \eta}{d x^{3}}+3 \eta \frac{d \eta}{d x}-\left(F^{2}-1\right) \frac{d \eta}{d x}=S f(\eta)
$$

We now make the transformation

$$
\eta=H+\frac{1}{3}\left(F^{2}-1\right)
$$

which gives

$$
\frac{1}{3}\left(d^{3} H / d x^{3}\right)+3 H(d H / d x)=S g(H) .
$$


The cnoidal solutions then satisfy

$$
\frac{1}{3}\left(d^{3} H / d x^{3}\right)+3 H(d H / d x)=0,
$$

and we can integrate twice to get

$$
\frac{1}{3}(d H / d x)^{2}=-H^{3}+A H+B,
$$

where $A$ and $B$ are constants of integration. Physically $A$ and $B$ are related in a complicated fashion to the energy and momentum flow defined earlier. It will be shown later that for certain well-defined values of $A$ and $B$ there are curves of constant $A$ and $B$ in the $\left(H^{\prime}, H\right)$ phase plane which are closed. It is for these values of $A$ and $B$ that periodic solutions of Eq. (3.1.4) exist.

There is, in fact, a doubly infinite family of cnoidal oscillations. The solution of those members of the family which neither gain nor lose energy or momentum over one wavelength is determined by the dissipation terms. It will be these particular cnoidal solutions that will be our approximation to the periodic solutions of Eq. (3.1.1).

We can integrate Eq. (3.1.5) again in terms of the Jacobi elliptic functions with solution

$$
H_{c}=\alpha+(\beta-\alpha) C_{n}^{2}\left[A_{1}\left(x+x_{0}\right), k\right]
$$

where $A_{1}, \alpha, \beta$, and $k$ are constants given by

$$
\begin{aligned}
B+A H-H^{3}= & (\beta-H)(H-\alpha)(H+\alpha+\beta), \quad \beta \geqq \alpha \geqq-\alpha-\beta, \\
& A_{1}^{2}=\frac{3}{4}(2 \beta+\alpha), \quad k^{2}=(\beta-\alpha) /(2 \beta+\alpha),
\end{aligned}
$$

and $k$ is the modulus of the elliptic function. Thus we can write $H$ in the form

$$
H=H_{c}\left(x+x_{0}, A, B\right),
$$

where the new constant of integration $x_{0}$ is the phase, and where the subscript $c$ is to emphasize that $H_{c}$ is a solution of the cnoidal approximation (3.1.4).

To obtain a solution of (3.1.1) we can use the method of variation of parameters and let $x_{0}, A$, and $B$ be functions of $x$. The dependence of $x_{0}, A$, and $B$ are then given by the equations

$$
\begin{aligned}
& \frac{d x_{0}}{d x}=-2 S\left(\frac{\partial H_{c}}{\partial A}-H_{c} \frac{\partial H_{c}}{\partial B}\right) g /\left(\partial H_{c} / \partial \phi\right), \\
& \frac{d A}{d x}=2 S g, \quad \frac{d B}{d x}=-2 S H_{c} g .
\end{aligned}
$$

The solution of the above equations using the explicit solution (3.1.6) would be an exact solution of Eq. (3.1.1). This is, of course, impossible. However, since we have surgested that for small $S$ the effect of the dissipation will be to slowly vary the shape of the cnoidal oscillations, it is only the overall change in $x_{0}, A$, and $B$ that we require, so we replace the right-hand side of Eq. (3.1.9) by the average over a wavelength. Thus

$$
\begin{aligned}
& \frac{d x_{0}}{d x}=-2 S \overline{\left(\frac{\partial H_{c}}{\partial A}-H_{c} \frac{\partial H_{c}}{\partial B}\right) g /\left(\partial H_{c} / \partial \phi\right),} \\
& \frac{d A}{d x}=2 S \bar{g}, \quad \frac{d B}{d x}=-2 S \overline{g H_{c}} .
\end{aligned}
$$


These equations are considerably simpler in that there is no explicit $x$-dependence and a solution is feasible. Also, since an average over a wavelength is independent of the phase, the last two equations suffice to determine $A(x)$ and $B(x)$. Thus the phase drops out of the problem and we are left with the two equations

$$
d A / d x=2 S \tilde{g}=2 S g_{1}(A, B),
$$

and

$$
d B / d x=-2 S \overline{H_{c} g}=2 S g_{2}(A, B) .
$$

We are interested in values of $A$ and $B$, say $A_{0}$ and $B_{0}$, which make

$$
g_{1}\left(A_{0}, B_{0}\right)=g_{2}\left(A_{0}, B_{0}\right)=0 .
$$

We can solve Eqs. (3.1.11) and (3.1.12) directly, but it is instructive to note that the solutions of Eqs. (3.1.13) are the singular points in the $(A, B)$ plane. This suggest that the structure of the solutions in the $(A, B)$ plane will be of interest. For example, if the singular point $\left(A_{0}, B_{0}\right)$ is a stable singular point we would expect to find the corresponding cnoidal wave train in a numerical integration of Eq. (3.1.1), whereas if the singular point was an unstable one we would not expect to find the corresponding cnoidal solution.

3.2 The structure of the $\left(H^{\prime}, H\right)$ and $(A, B)$ phase planes. The cnoidal curves, or curves of constant $A$ and $B$, in the $\left(H^{\prime}, H\right)$ phase plane are given by

$$
\frac{1}{3}(d H / d x)^{2}=-H^{3}+A H+B .
$$

We must discuss how the "cnoidal" curves depend on $B$. The points of equilibrium are given by

$$
d^{2} H / d x^{2}=0, \text { or }-3 H^{2}+A=0,
$$

so that for $A<0$ there are no positions of equilibrium while for $A>0$ there are two, given by $H= \pm(\bar{A} / 3)^{1 / 2}$.

If $H_{0}$ is a position of equilibrium, then the character of the equilibrium point may be investigated by studying solutions of the form $H=H_{0}+h$ for small $h$. The equation for $h^{\prime \prime}$ then becomes

$$
\frac{1}{3} h^{\prime \prime}=-3 H_{0} h .
$$

We see from this that the point $H_{0}=(A / 3)^{1 / 2}$ is a center, while the point $H_{0}=$ $-(\bar{A} / 3)^{1 / 2}$ is an unstable saddle point.

When $B$ takes the value $B_{0}=-2\left(A^{3} / 27\right)^{1 / 2}$ the curve in the neighborhood of $H=$ $(\bar{A} / 3)^{1 / 2}$ is just the single point $H=(\bar{A} / 3)^{1 / 2}$. For values of $B$ just greater than $B_{0}$ the curves are closed ovals. If $A$ is fixed and $B$ increases, then the size of the loop increases until $B=+2\left(\bar{A}^{3} / 27\right)^{1 / 2}$. This is the limiting curve which passes through the singular point $H=-(\bar{A} / 3)^{1 / 2}$. As $B$ increases still further, the curve is no longer closed (see Fig. 2). For bounded oscillatory solutions we must be within the loop of this limiting curve which divides the closed curves from the unbounded curves. This limiting curve corresponds to the solitary wave and represents an asymptotic approach to the unstable equilibrium point.

For solution curves corresponding to those inside this loop we must have the roots of the cubic on the right-hand side of Eq. (3.1.5) real. 


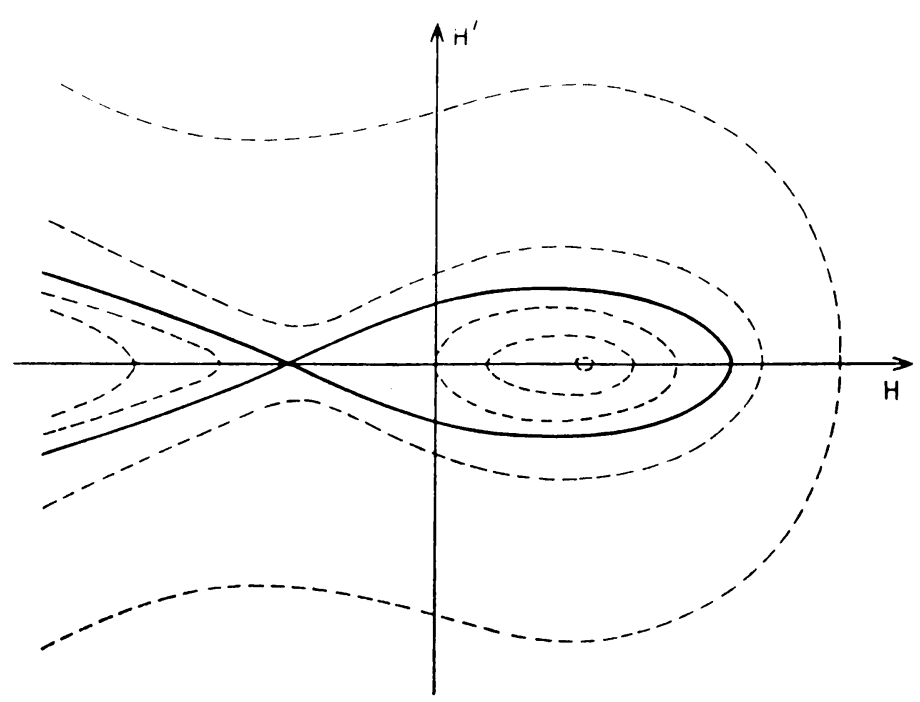

FIG. 2. The $\left(H^{\prime}, H\right)$ phase plane for $A$ positive

This gives the condition

$$
A^{3} / 27>B^{2} / 4 \text {. }
$$

This splits the $(A, B)$ plane up into two regions bounded by the curve $A^{3} / 27=B^{2} / 4$. Only points right of this curve (see Fig. 3) correspond to bounded solutions in the $\left(H^{\prime}, H\right)$ phase plane. Points of stable equilibrium in the $(A, B)$ plane correspond to a train of uniform cnoidal waves (for solutions see Eq. (3.1.6)) varying from the solitary wave on the upper boundary $\left(B=+2\left(\bar{A}^{3} / 27\right)^{1 / 2}\right.$ to uniform flow on the lower boundary $\left(B=-2\left(\bar{A}^{3} / 27\right)^{1 / 2}\right.$.

3.3 The solution in the $(A, B)$ plane with Chézy resistance. We remarked earlier that the solution with Chézy resistance did not seem to settle down to a regular oscillation

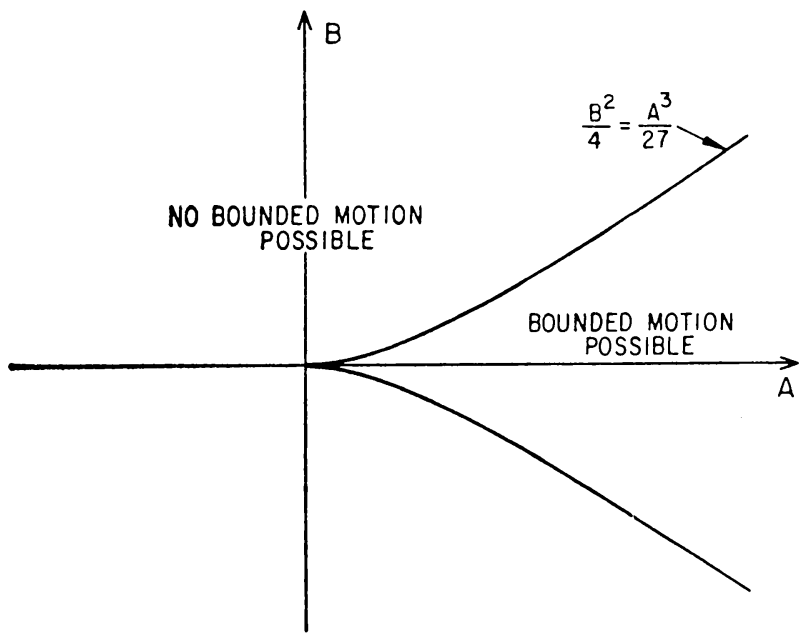

Fig. 3. The $(A, B)$ plane 
even when integration was carried out over a large distance. Although the resulting averaged equations (3.1.11) and (3.1.12)) still need to be integrated numerically, the need to integrate over large distances is removed by the method of solution outlined in Sec. 3.1. This is because the small parameter $s$ can be scaled out of the equations and the step length taken is effectively $s^{-1}$ times as great as the step length required for the full Eq. (3.1.1).

For this problem the resistance term becomes

$$
g(H)=\left(H_{2}-H\right)\left(H_{1}+H\right)
$$

where $H_{1}=\frac{1}{3}\left(F^{2}-1\right)$ and $H_{2}=\eta_{2}-\frac{1}{3}\left(F^{2}-1\right)$. The averaged equations are then

$$
d A / d x=2 s\left(H_{1} H_{2}+\left(H_{2}-H_{1}\right) \bar{H}-\vec{H}^{2}\right),
$$

and

$$
d B / d x=-2 s\left(H_{1} H_{2} \vec{H}+\left(H_{2}-H_{1}\right) H^{2}-\vec{H}^{3}\right) .
$$

The average of a power of $H$ is fairly easily obtained in terms of $k$ and either $\alpha$ or $\beta$. The first three are

$$
\begin{aligned}
\vec{H}=\beta\left[3 E / K-2+k^{2}\right] /\left(1+k^{2}\right)=l \beta . & \\
\bar{H}^{2}=\beta^{2}\left[1-k^{2}+k^{4}\right] /\left(1+k^{2}\right)^{2}=m \beta^{2} . & \left.\quad+\left(4-2 k^{2}\right)^{3}\right\} /\left(1+k^{2}\right)^{3}=n \beta^{3},
\end{aligned}
$$

where $l, m$, and $n$ are functions of $k^{2}$ only, and $K$ and $E$ are the complete elliptic integrals of the first and second kind, respectively. This gives

$$
d A / d x=2 s\left[H_{1} H_{2}+\left(H_{2}-H_{1}\right) \beta l-\beta^{2} m\right],
$$

and

$$
d B / d x=-2 s\left[H_{1} H_{2} l+\left(H_{2}-H_{1}\right) \beta m-\beta^{2} n\right] .
$$

The equilibrium points of the $(A, B)$ phase plane are given by

$$
d A / d x=d B / d x=0 .
$$

Hence $\beta$ must satisfy

$$
H_{1} H_{2}+\left(H_{2}-H_{1}\right) \beta l-\beta^{2} m=H_{1} H_{2} l+\left(H_{2}-H_{1}\right) \beta m-\beta^{2} n=0 .
$$

The two quadratic equations have equal roots when $k$ is given by the solution of the equation

$$
\left(m-l^{2}\right)\left(m^{2}-n l\right) /(n-m l)^{2}=H_{1} H_{2} /\left(H_{2}-H_{1}\right)^{2} .
$$

The solution for $\beta$ is then

$$
\beta=\left(H_{2}-H_{1}\right)\left(m-l^{2}\right) /(n-m l) .
$$

We also get a solution when one quadratic equation is a multiple of the other; this occurs when

$$
m-l^{2}=n-m l=m^{2}-n l=0 .
$$


The latter case occurs (1) for $k=0$ when $l=m=n=1$, the solution for $\beta$ is then $\beta=H_{2}$ or $-H_{1} ;(2)$ for $k=1$ when $l=\frac{1}{2}, m=\frac{1}{4}, n=-\frac{1}{8}$; the solution for $\beta$ is then $\beta=2 H_{1}$ or $-2 H_{2}$.

To study the character of these equilibrium points it is now easier to work in terms of $\alpha$ and $\beta$, so we substitute for $A$ and $B$ by

$$
B=-\alpha \beta(\alpha+\beta),
$$

and

$$
A=\alpha^{2}+\alpha \beta+\beta^{2} .
$$

The character of the equilibrium points $\left(\alpha_{0}, \beta_{0}\right)$ may be investigated by studying solutions of the form $\alpha=\alpha_{0}+\delta, \beta=\beta_{0}+\gamma$.

For the equilibrium point $\beta=H_{2}, \alpha_{0}=H_{2}$, Eqs. (3.3.7) and (3.3.8) become

$$
\delta^{\prime}=-s\left(\frac{H_{2}+H_{1}}{12 H_{2}}\right)[\delta-3 \gamma]
$$

and

$$
\gamma^{\prime}=-s\left(\frac{H_{2}+H_{1}}{12 H_{2}}\right)[-3 \delta+\gamma] .
$$

Hence this gives an unstable saddle point in the $(B, A)$ phase plane with singular directions

$$
d B / d A=-H_{2} \text { and } 2 H_{2} .
$$

For the equilibrium point $\beta=2 H_{1}, \alpha=-H_{2}$ the wavelength becomes infinite. In this case the averaging breaks down and the form of the solutions is not given by the solution of Eqs. (3.3.7) and (3.3.8). However, the behavior of the solution curves in the neighborhood of the singular point is of interest since we need to know whether the point is a stable or an unstable point. We can determine this by studying the limit of $(d B / d x) /(d A / d x)$ as $\beta \rightarrow 2 H_{1}, \alpha \rightarrow-H_{1}$, so as to find the singular directions (if there are any).

This, in fact, gives two cases:

(1) $H>1 \cdot 4 H_{1}$. In this case we get a saddle point.

(2) $H_{2}<1 \cdot 4 H_{1}$. In this case the singular point is a stable node.

The two singular directions are in both cases

$$
d B / d A=H_{1} \text { or }-\left(H_{2}-\left(9 H_{1} / 5\right)\right) H_{1} /\left(H_{2}-H_{1}\right) .
$$

The diagrams of the solution curves in the neighborhood of the singular points are given in Figs. 4 and 5.

By considering the transformation $H_{2} \leftrightarrow H_{1}$ we can show that when $H_{1}<0$ the singular point given by $k=0, \beta=-2 H_{1}$, is a saddle point. By making the same transformation we can show that when $H_{2}<0$ the singular point given by $k=1, \beta=-2 H_{2}$ is a saddle point.

The position of the third singular point depends on the solution of $k=k_{0}$ of Eq. (3.3.11).

The function $G(k)=\left(m-l^{2}\right)\left(m^{2}-l n\right) /(n-l m)^{2}$ is a monotonically increasing 


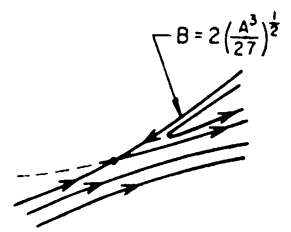

FIG. 4. $H_{2}>1.4 H_{1}$. A sketch of the solution curves near the singular points corresponding to $\beta=2 H_{1} k=1$.

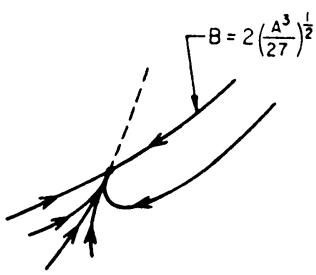

Fia. 5. $H_{2}>1.4 H_{1}$. A sketch of the solution curves for the singular points corresponding to $\beta=2 \mathrm{H}_{1} k=1$.

function of $k$ in the range $(0,1)$ and varies from -0.25 when $k=0$ to 8.75 when $k=1$ (see Fig. 6). Therefore, the value of $H_{1} H_{2} /\left(H_{2}-H_{1}\right)^{2}$ must lie between -0.25 and 8.75 for this singular point to exist. We also require $\beta>0$ and since $\left(m-l^{2}\right) /(n-l m)>0$, then $H_{2}>H_{1}$. Therefore, this singular point exists for all values of $H_{1} / H_{2}$ in the range $-1<H_{1} / H_{2}<5 / 7$. The location of the singular point in the $(A, B)$ plane is given in Fig. 7.

Thus when $H_{2}>1.4 H_{1}$ we have three singular points, and when $H_{2}<1.4 H_{1}$ we have only two. For the second case the solution curves can be drawn immediately (see Fig. 8). It can be seen that a solution starting from $H=H_{2}$ at $x=-\infty$ and ending at $H=-H_{1}$ as $x \rightarrow+\infty$ does exist on this theory. However, due to the inadequacies of the averaging technique along and near the line $B^{2} / 4=A^{3} / 27$ it is impossible to say whether this solution represents a good approximation to the solution of the full equation

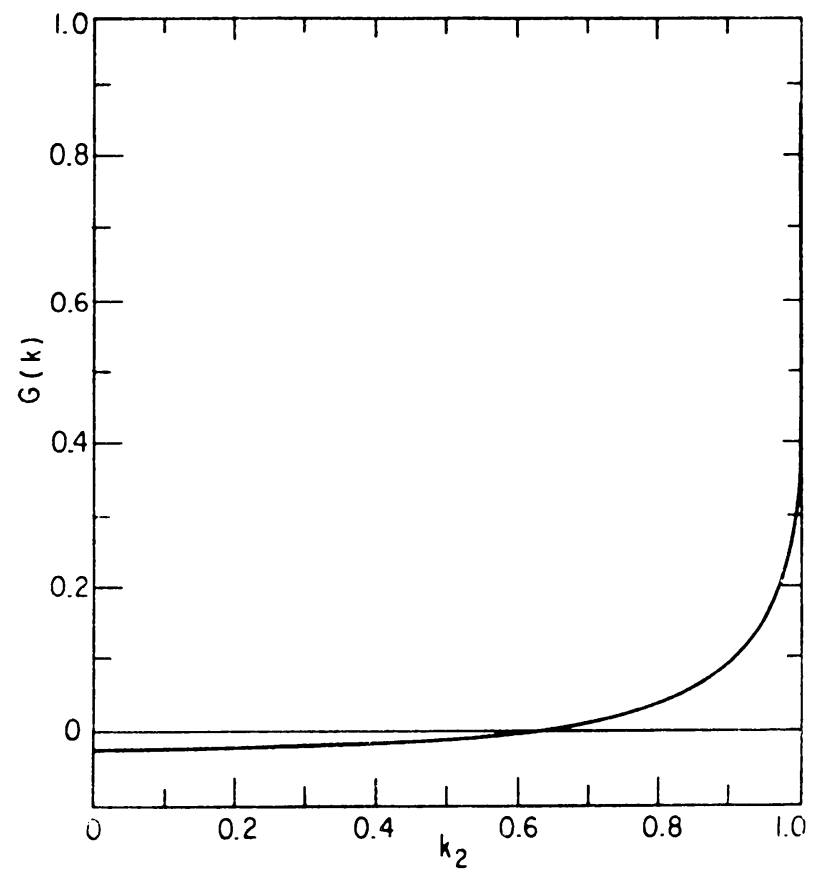

FIG. 6. A graph of the function $G(k)=\frac{\left(m-l^{2}\right)\left(m^{2}-n l\right)}{(n-l n)^{2}}$ 


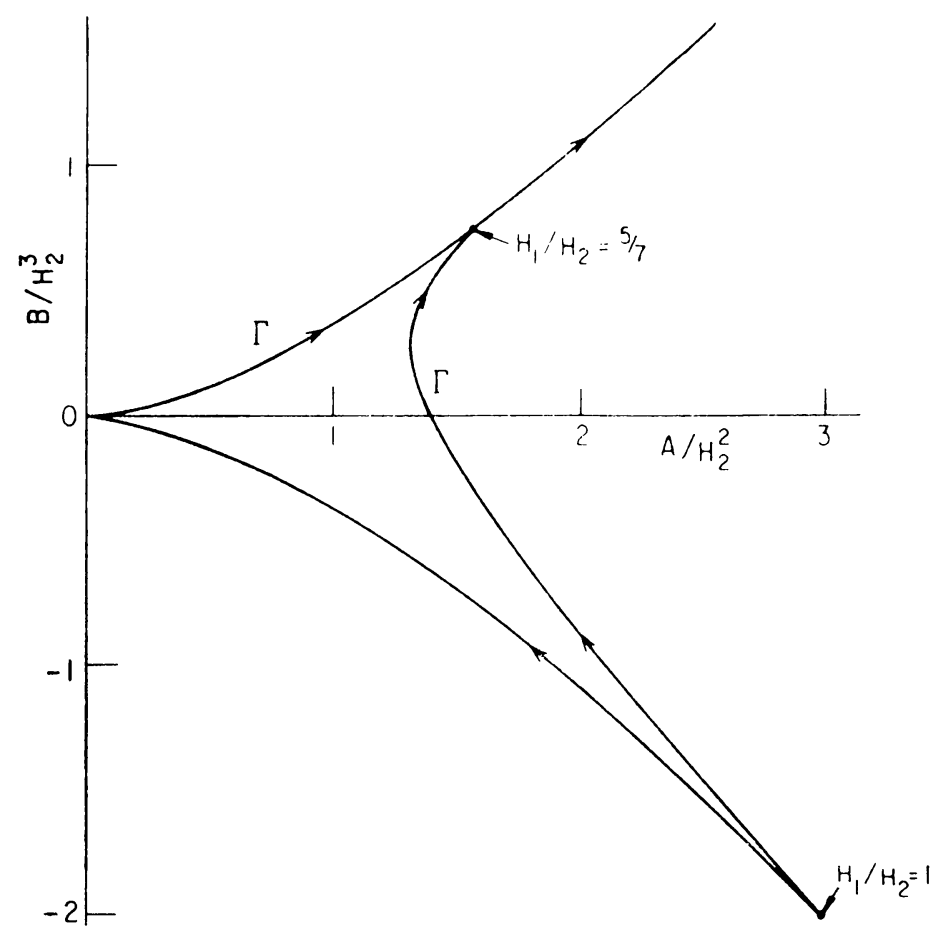

Fia. 7. A sketch of the $(A, B)$ plane. The locus $\Gamma$ of values $\left(A_{0}, B_{0}\right)$ corresponding to steady periodic solutions is shown.

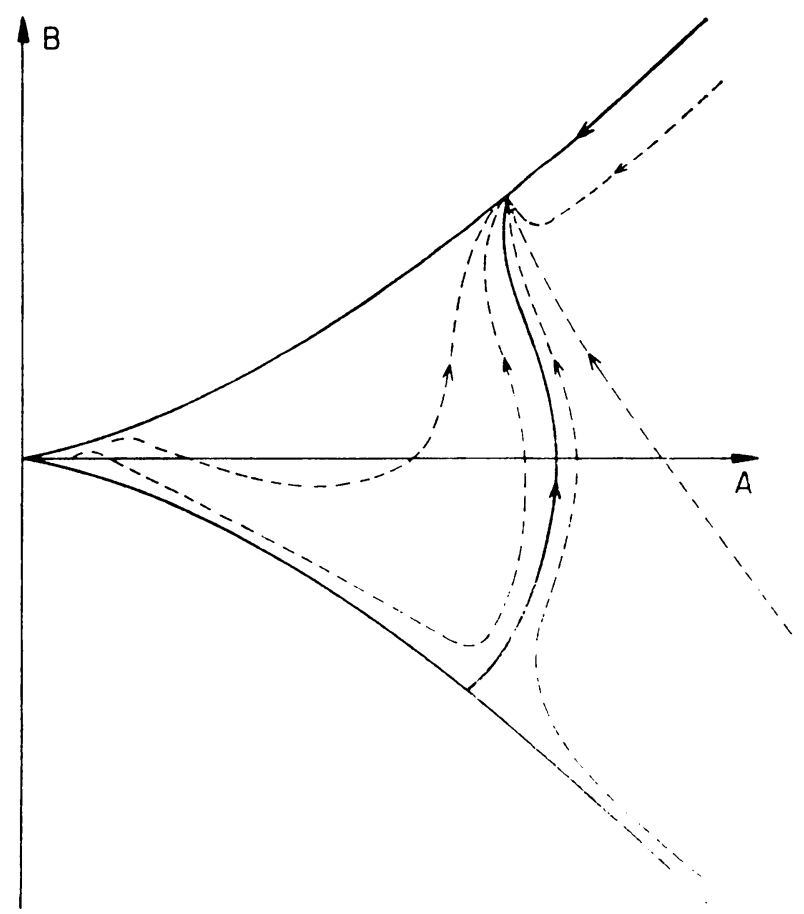

FIG. 8. Solution curves for $H_{2}<1.4 H_{1}$. 


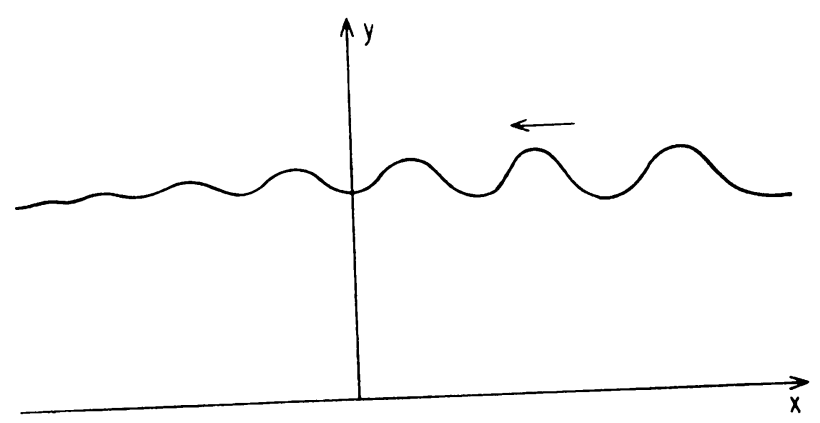

FIG. 9. Representation of the negative bore solution

as we approach the singular point as $x \rightarrow \infty$. The exact solution might quite possibly be carried through the singular point into the region $B^{2} / 4>A^{3} / 27$ and then the solution for $H$ will tend to $\infty$. Certainly numerical integration of the full equation exhibits this behavior when $\mathrm{H}_{2}<1.4 \mathrm{H}$. If this solution does exist it represents a steady bore of negative strength, and is shown in Fig. 9.

For the case $H_{2}>1.4 H_{1}$ we have three singular points. The two on the boundary $B^{2} / 4=A^{3} / 27$ are unstable. All that remains is to determine the character of the third singular point. Since the solution of Eq. (3.3.11), which gives the location of the third singular point, can only be done numerically, the best method of investigation into the character of this point is by studying the numerical solutions of Eqs. (3.3.2) and (3.3.3). The numerical solutions for the solution curves in the $(A, B)$ plane are given for various

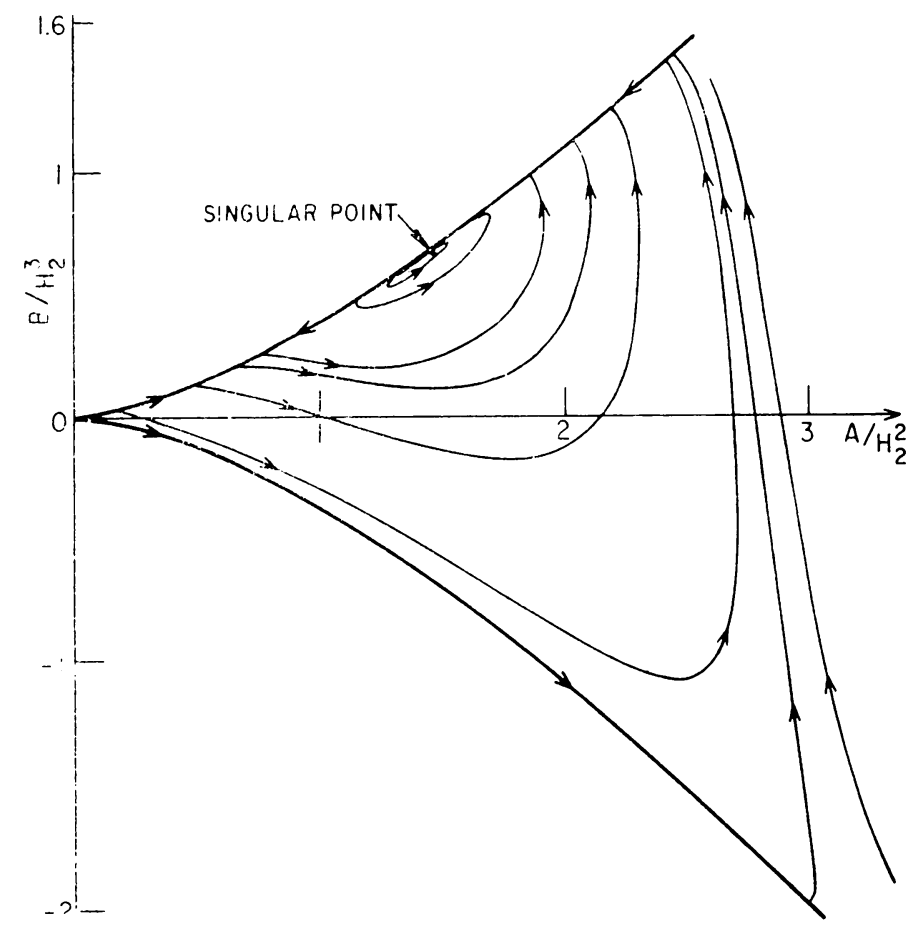

Fici. 10. Solution curves in the $(A, B)$ plane for $H_{1} / H_{2}=\frac{1}{2}$. 


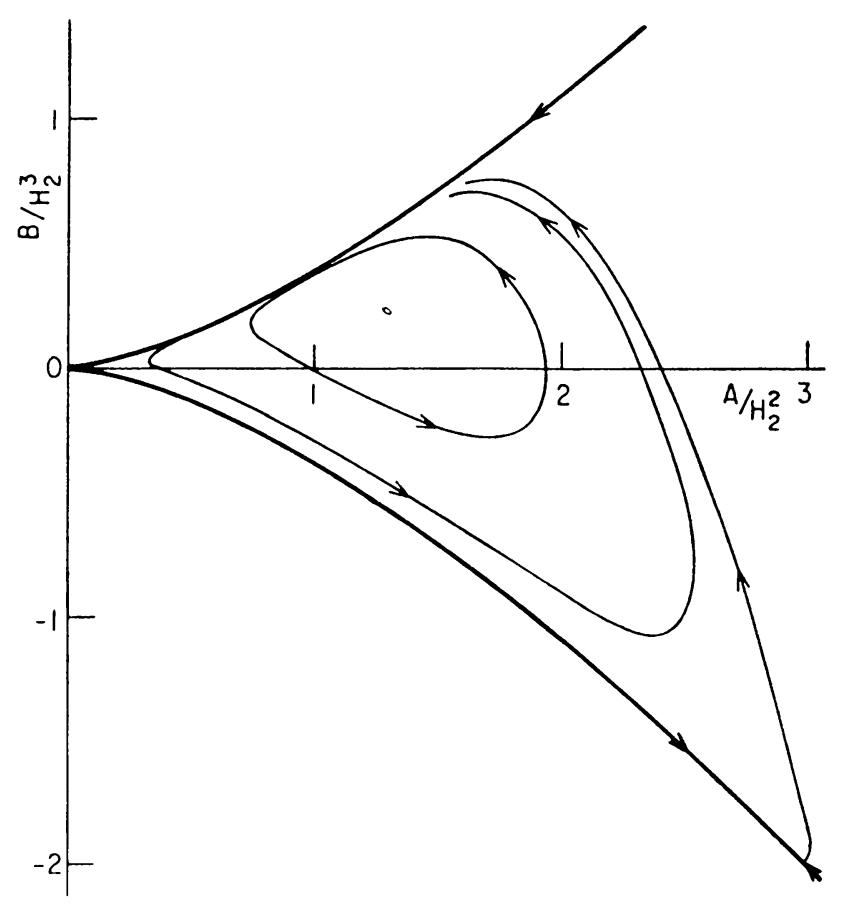

FIG. 11. Solution curves in the $(A, B)$ plane for $H_{1} / H_{2}=0$.

values of $H_{1} / H_{2}$ (see Figs. 10, 11, and 12). These solutions possess some remarkable properties. When the singular point $\left(A_{0}, B_{0}\right)$ is far from the boundary the solution curves in the neighborhood of $\left(A_{0}, B_{0}\right)$ seem, to a very good approximation, to be elliptical; this suggests that $\left(A_{0}, B_{0}\right)$ is a center on a linear theory. Even at large distances from $\left(A_{0}, B_{0}\right)$ the numerical solution still seemed to be a closed curve. Thus the numerical analysis tells us that we have a focal point, but does not tell us whether it is stable or unstable. In fact, the supposition that $A_{0}, B_{0}$ is a center on a linear theory can be proved analytically if we use the fact that we know from the numerical results that $\left(A_{0}, B_{0}\right)$ is a focal point. (See Appendix.) Therefore, since all curves have to tend to some region around $\left(A_{0}, B_{0}\right)$, on an exact theory $\left(A_{0}, B_{0}\right)$ must either be a stable focal point or an unstable focal point with a limit cycle surrounding it. It is also to be noted that the density of solution curves becomes very high near the boundary where the averaging becomes suspect.

4. Discussion of the solutions. We have shown that only in the case when $H_{2}>0$ and $H_{2}>1.4 H_{1}$ does an acceptable bounded solution exist. For $H_{1}>0$ we do in fact have two bounded solutions, one that starts as uniform supercritical flow at $r=-\infty$ and tends to a uniform train of waves at $x=+\infty$. This represents a solution for a steady bore running down hill and is the type of solution for which we have been looking (see Fig. 13). The other solution is one that starts as uniform subcritical flow at $x=-\infty$. When $H_{1}<0$ the second type of solution is the only one that exists.

We can also explain the reasons for the unexpected numerical results obtained from integration of the full equation. As $H_{1} / H_{2}$ increases from -1 to $5 / 7$ the singular point moves closer to the upper boundary. Then the solution curves run extremely close together and with numerical errors the solution easily moves from one curve to another 


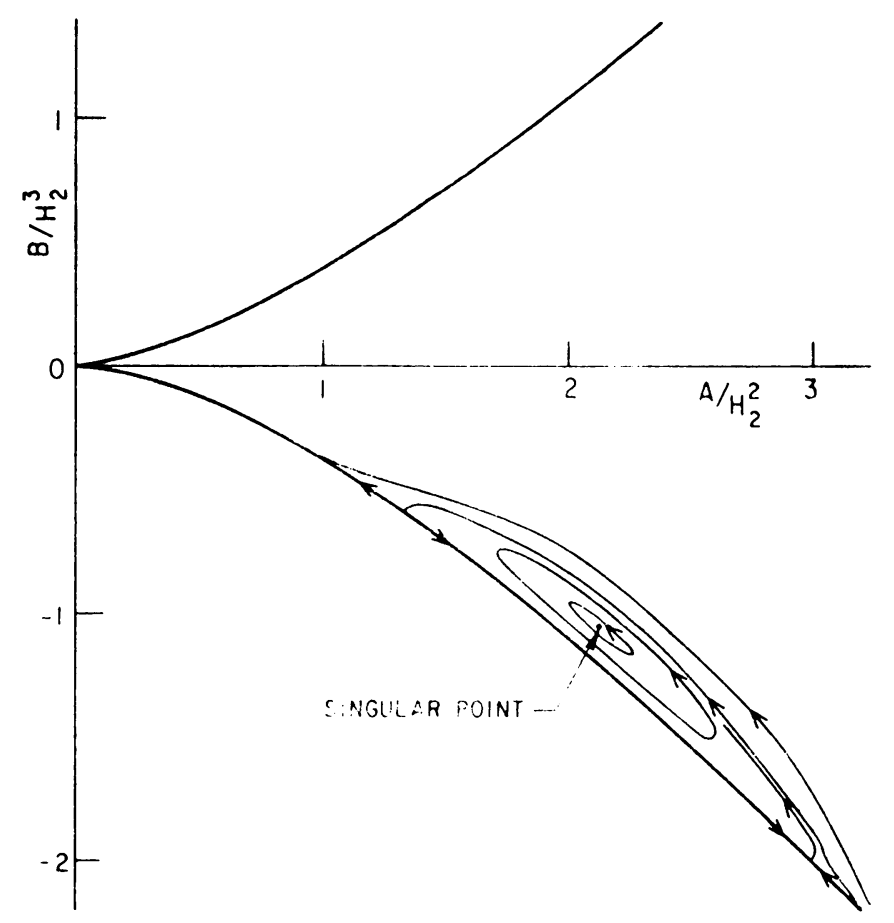

Fra. 12. Solution curves in the $(A, B)$ plane for $H_{1} / H_{2}=-\frac{2}{3}$.

in this region near the boundary, so that no regularity in the solution would be observed. Also, since $\left(A_{0}, B_{0}\right)$ is a center on a linear theory, the convergence of the solution to the point $\left(A_{0}, B_{0}\right)$ is very slow, so that integration would be broken off long before a steady oscillation was reached, especially if $S$ is taken to be very small.

Appendix. An analytical investigation into the character of the third singular point. The numerical results of the averaged equations suggest that the third singular point $\left(A_{0}, B_{0}\right)$ is a center for all values of $H_{1} / H_{2}$ in the range $(-1,5 / 7)$. Although a complete analytical investigation into the character of $\left(A_{0}, B_{0}\right)$ is not possible, we can show that $\left(A_{0}, B_{0}\right)$ is a center without actually solving Eqs. (3.3.11) to find $A_{0}$ and $B_{0}$. We can

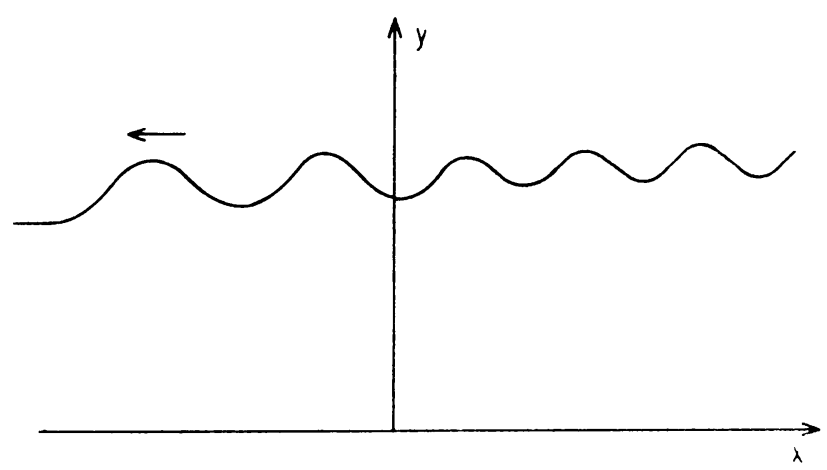

FIG. 13. Representation of the bore solution. 
do this by introducing a generating function $W$, defined by

$$
W(A, B)=\oint\left(H_{c}^{\prime}\right)^{2} d x=\oint H_{c}^{\prime} d H
$$

where the integral is taken around the cnoidal curves in the $\left(H^{\prime}, H\right)$ phase plane given by $A$ and $B$ and Eq. (3.1.5).

Using this function we can express $d A / d x$ and $d B / d x$ in terms of $W$ and its derivatives with respect to $A$ and $B$.

We recall that Eq. (3.1.5) is

$$
\frac{1}{3}(\partial H / \partial x)^{2}=-H^{3}+A H+B,
$$

with the help of this equation we can calculate the derivatives of $W$ with respect to $A$ and $B$.

Thus

$$
\partial W / \partial B=\frac{\partial}{\partial B} \oint \sqrt{ } 3\left(-H^{3}+A H+B\right)^{1 / 2} d H=3 \lambda / 2
$$

and

$$
\frac{\partial W}{\partial A}=\frac{\partial}{\partial A} \oint \sqrt{ } 3\left(-H^{3}+A H+B\right)^{1 / 2} d H=\frac{3 \lambda}{2} \bar{H}
$$

where $\lambda$ is the wave length of the cnoidal oscillation. Since the average value of $d H / d x^{2}$ is zero, we have

$$
\frac{1}{\lambda} \oint \frac{d^{2} H}{d x^{2}}=0=\frac{1}{\lambda} \int_{0}^{\lambda}\left(-3 H^{2}+A\right) d x
$$

Therefore

$$
\bar{H}^{2}=\frac{1}{3} A \text {. }
$$

Finally the average value of $H^{3}$ is found from equation (A.1) as

$$
\bar{H}^{3}=A \bar{H}+B-\frac{1}{3} W / \lambda \text {. }
$$

Eqs. (3.3.2) and (3.3.3) then become

$$
\begin{aligned}
d A / d X & =(4 \epsilon / 3 \lambda)\left[W_{B} H_{1} H_{2}+\left(H_{2}-H_{1}\right) W_{A}+\frac{1}{3} A W_{B}\right]=g_{1}(A, B), \\
d B / d X & =(-4 \epsilon / 3 \lambda)\left[H_{1} H_{2} W_{A}+\left(H_{2}-H_{1}\right) \frac{1}{3} A W_{B}+\frac{1}{2} W-A W_{A}-B W_{B}\right] \\
& =g_{2}(A, B) .
\end{aligned}
$$

The condition for $A_{0} B_{0}$ to be a center is

$$
\left(\partial g_{1} / \partial A\right)_{A_{0} B_{0}}+\left(\partial g_{2} / \partial B\right)_{A_{0} B_{0}}=0,
$$

and

$$
\left(\frac{\partial g_{1}}{\partial A}\right)_{A \circ B_{0}}\left(\frac{\partial g_{2}}{\partial B}\right)_{A_{\diamond} B_{0}}-\left(\frac{\partial g_{1}}{\partial B}\right)_{A_{\diamond} B_{0}}\left(\frac{\partial g_{2}}{\partial A}\right)_{A_{\diamond} B_{0}}>0 .
$$

(A.11) is the condition for a focal point and (A.10) tells us that the focal point is a center. We now appeal to the results of the numerical integration to say that the 
second condition is satisfied since the solution curves near $\left(A_{0} B_{0}\right)$ are approximately ellipses. From Eqs. (A.8) and (A.9) the condition for a center is thus

$$
\frac{4 \epsilon}{3 \lambda}\left[\left(H_{2}-H_{1}\right)\left(\frac{\partial^{2} W}{\partial A^{2}}-\frac{A}{3} \frac{\partial^{2} W}{\partial B^{2}}\right)+\left(\frac{1}{6} \frac{\partial W}{\partial B}+B \frac{\partial^{2} W}{\partial B^{2}}+\frac{2 A}{3} \frac{\partial^{2} W}{\partial_{B} \partial_{A}}\right)\right]=0 .
$$

Partial differentiation of Eq. (A.1) gives

$$
\begin{aligned}
\frac{\partial^{2} W}{\partial A^{2}}-\frac{A}{3} \frac{\partial^{2} W}{\partial B^{2}} & =\frac{\sqrt{ } 3}{4} P \oint \frac{\frac{A}{3}-H^{2}}{\left(-H^{3}+A H+B\right)^{3 / 2}} d H \\
& =-\frac{1}{2} P \oint \frac{d}{d H}\left(\frac{1}{H^{\prime}}\right) \\
& =0,
\end{aligned}
$$

and

$$
\begin{aligned}
\frac{1}{6} \frac{\partial I V}{\partial B}+ & B \frac{\partial^{2} W}{\partial B^{2}}+\frac{2}{3} \frac{\partial^{2} W}{\partial_{A} \partial_{B}} \\
& =\frac{\sqrt{ } 3}{2} \oint \frac{\frac{1}{6}-d H}{\left(-H^{3}+A H+B\right)^{1 / 2}}-\frac{\sqrt{3}}{4} P \oint \frac{\left(B+\frac{2}{3} A H\right) d H}{\left(-H^{3}+A H+B\right)^{1 / 2}} \\
& =-\frac{1}{2} P \oint \frac{d}{d H}\left(\frac{H}{H^{\prime}}\right) \\
& =0
\end{aligned}
$$

where $P \oint$ denotes the fact that we have taken the Cauchy principle value of the integral. Hence condition (A.10) is satisfied for all values of $H_{1} / H_{2}$ so that the singular point is always a center.

\section{REFERENCES}

[1] G. B. Airy, "Tides and waves," in Encyclopaedia metropolitana, London (1845)

[2] T. B. Benjamin and M. J. Lighthill, On cnoidal waves and bores, Proc. Roy. Soc. London Ser. A224, 448-460 (1954)

[3] J. Boussinesq, Essai sur la thcorie des eaux courants, Mém. Pres. Acad. Sci. Paris 23 (1877)

[4] W. Chester, Proc. Roy. Soc. London Ser. A 306, 5 (1968)

[5] R. F. Dressler, Mathematical solution of the problem of roll-waves in inclined open channels, Comm. Pure Appl. Math. 2, 149-194 (1949)

[6] H. Favre, Etude thíorique et experimentale des ondes de translation dans les canaux découvents, Dunod, Paris, 1935

[7] G. H. Keulegan and G. W. Patterson, Mathematical theory of irrotational translation waves, J. Research Nat. Bur. Standards 24, 47-101 (1940)

[8] D. J. Korteweg and G. De Vries, Philos. Mag. (5) 39, 422 (1895)

[9] R. Lemoine, Sur les ondes positives de translation dans les canaux et sur le ressaut ondule de faible amplitude, La Houille Blanch 2, Grenoble, France (1948)

[10] M. J. Lighthill and G. B. Whitham, On kinematic waves. I: Flood movement in long rivers, Proc. Roy. Soc. London Ser. A 229, 281-316 (1955)

[11] Lord Rayleigh, Philos. Mag. (5) 1, 247 (1876); Proc. Roy. Soc. London Ser. A 90, 344 (1914)

[12] S. J. Russell, Rep. Brit. Assn. 417 (1837) Rep. Brit. Assn. p. 311 (1844)

[13] J. A. Sandover and T. D. Taylor, Experiments on surge waves, Water Power 9, 418 (1957)

[14] J. A. Sandover and O. C. Zienkiewicz, La Houille Blanch 17, 443, Grenoble, France (1962)

[15] H. A. Thomas, Hydraulics of flood movement in rivers, Carnegie Institute of Technology, Pittsburg, 1937 